\title{
Graze: A program to analyze recordings of the jaw movements of ruminants
}

\author{
S. MARK RUTTER \\ Institute of Grassland and Environmental Research, North Wyke, England
}

\begin{abstract}
Graze is a user friendly, Microsoft Windows 95/NT program for analyzing jaw movement recordings taken with the IGER Behavior Recorder. The program displays a plot of the jaw movement amplitude (vertical axis) against time (horizontal axis). Individual jaw movements can be automatically identified, using amplitude and frequency criteria specified by the user. Bouts of jaw movements can then be analyzed and are automatically identified as either grazing or ruminating. Behaviors other than grazing and ruminating (such as drinking or eating supplements) can also be identified and marked by the user. Identified jaw movements and bouts of behavior are superimposed on the jaw data display. Finally, the user can perform a bout analysis, which discriminates between bites and chews during eating and generates a summary file.
\end{abstract}

In order to improve our understanding of the detailed mechanics of ruminant foraging behavior, we need to be able to make precise measurements of the grazing behavior of free-ranging animals (Hodgson, 1977). Penning (1983) developed a jaw movement recording system that could be carried by sheep. The animal's jaw movements were sensed with a carbon-filled silicone tube placed around the sheep's muzzle. As the animal's jaws opened and closed, the sensor was stretched, and its electrical resistance changed. These changes in electrical resistance were recorded on a miniature analogue tape recorder carried on the sheep's back. Subsequently, the recorded signals were analyzed by a microcomputer to determine periods of eating and ruminating and the total numbers of jaw movements. Further development of the system (Penning, Steel, \& Johnson, 1984) allowed discrimination between bites and chews during grazing.

Although Penning's (1983) system was used in numerous grazing studies, the analogue recording system was prone to mechanical problems, and the cassette recorders were difficult to service, since they had become obsolete. Consequently, a replacement system was required. Rutter, Champion, and Penning (1997) reviewed the various grazing behavior recording techniques developed by other researchers and found that none provided a satisfactory replacement for Penning's system. Consequently, Rutter, Champion, and Penning developed their own computerbased recording system, known as the IGER Behavior Recorder. This article describes the functioning of the

Graze was developed as part of a project funded by the U.K. Ministry of Agriculture, Fisheries, and Food. The IGER Behavior Recorder and Graze are commercially available from Ultra Sound Advice Ltd. (details from the Website at www.ultrasoundadvice.co.uk). The author has a financial interest in these products. Correspondence concerning this article should be addressed to S. M. Rutter, Institute of Grassland and Environmental Research, North Wyke, Okehampton, Devon EX20 2SB, England (e-mail: mark.rutter@bbsrc.ac.uk). program (called Graze) that is used to analyze jaw movement recordings made with the IGER Behavior Recorder. Although designed for use in ruminant foraging behavior studies, the system can be used to study the jaw movements of any animal above a minimum size. Examples include changes in ruminating behavior associated with bovine spongiform encephalopathy in cattle or crib-biting stereotyped behavior in horses.

\section{METHOD}

\section{Data Collection and Storage}

The animal's jaw movement data are collected by using the IGER Behavior Recorder (Rutter, Champion, \& Penning, 1997), a brief description of which follows. A jaw movement sensor, identical to the one used by Penning (1983), is used to sense the animal's jaw movements. The signal from this sensor is linked via a filter to the analoguedigital converter on a small microcomputer. The signal is sampled 20 times a second and saved as an 8-bit integer. Consequently, measurements of jaw movement amplitudes can have values ranging from 0 to 255 and are given in an arbitrary unit of measurement. The jaw movement amplitude data are saved in Binary Behavior Data (.BIN) files. These consist of a 512-byte header, followed by one or more data blocks. The header contains various data, including the recorder number and the time and date when the recorder was started, when measurements started, and when measurements ended. The header also contains pointers and byte counts for the assorted data blocks that follow the header. Exact details of the Binary Behavior Data file format are given in Rutter, Champion, and Penning. (Note that the contents of bytes 328-331 are incorrectly labeled in Rutter, Champion, and Penning and should be labeled as "Interval between 1 bit walking sensor data.")

The IGER Behavior Recorder has the capability to record behaviors other than behavior related to jaw 
movement--for example, lying, standing, walking, and location-as described in Champion, Rutter, and Penning (1997) and Rutter, Beresford, and Roberts (1997). However, the current version of Graze will only analyze jaw movement data, as will be described in this article.

\section{Displaying Raw Jaw Movement Data}

After starting the Graze program, a Binary Behavior Data file can then be opened, using the Open Behavior File menu command. The main display area of the Graze window displays a plot of the jaw movement amplitude (vertical axis) against time (horizontal axis), as is shown in Figure 1. The relative scale of the vertical jaw movement amplitude axis remains fixed, ranging from an amplitude value of 0 at the bottom of the window to a value of 255 at the top of the window. The midpoint value of 128 (corresponding to the baseline signal on a correctly adjusted IGER Behavior Recorder) is shown by a thin, blue dotted line across the middle of the window.

The horizontal time scale can be changed by using the Scale menu, allowing the user to effectively zoom in and out of the recording. Minute boundaries are shown by thin, blue, vertical dotted lines at the appropriate position. The current horizontal scale can be judged by using the Scale box (as shown in Figure 1), which can be displayed by using the Show Scale Box menu command. The user can scroll through the data file, using the horizontal scroll bar at the bottom of the Graze window. As the user moves the mouse cursor across the main display window, the time at which the datapoint under the cursor was recorded is displayed at the top left-hand corner of the screen (following the word "Mouse"). The user can position a vertical, flashing cursor in the window by moving the mouse cursor to the appropriate position and then pressing the mouse button. The time position of the flashing cursor is given in the upper-left area of the window, followed by the interval between the flashing cursor and the mouse position (following the words "Cursor" and "Delta," respectively). This feature allows the user to accurately measure the time interval between events displayed in the main window. Finally, the user can view pertinent information about the data file (e.g., recorder num-

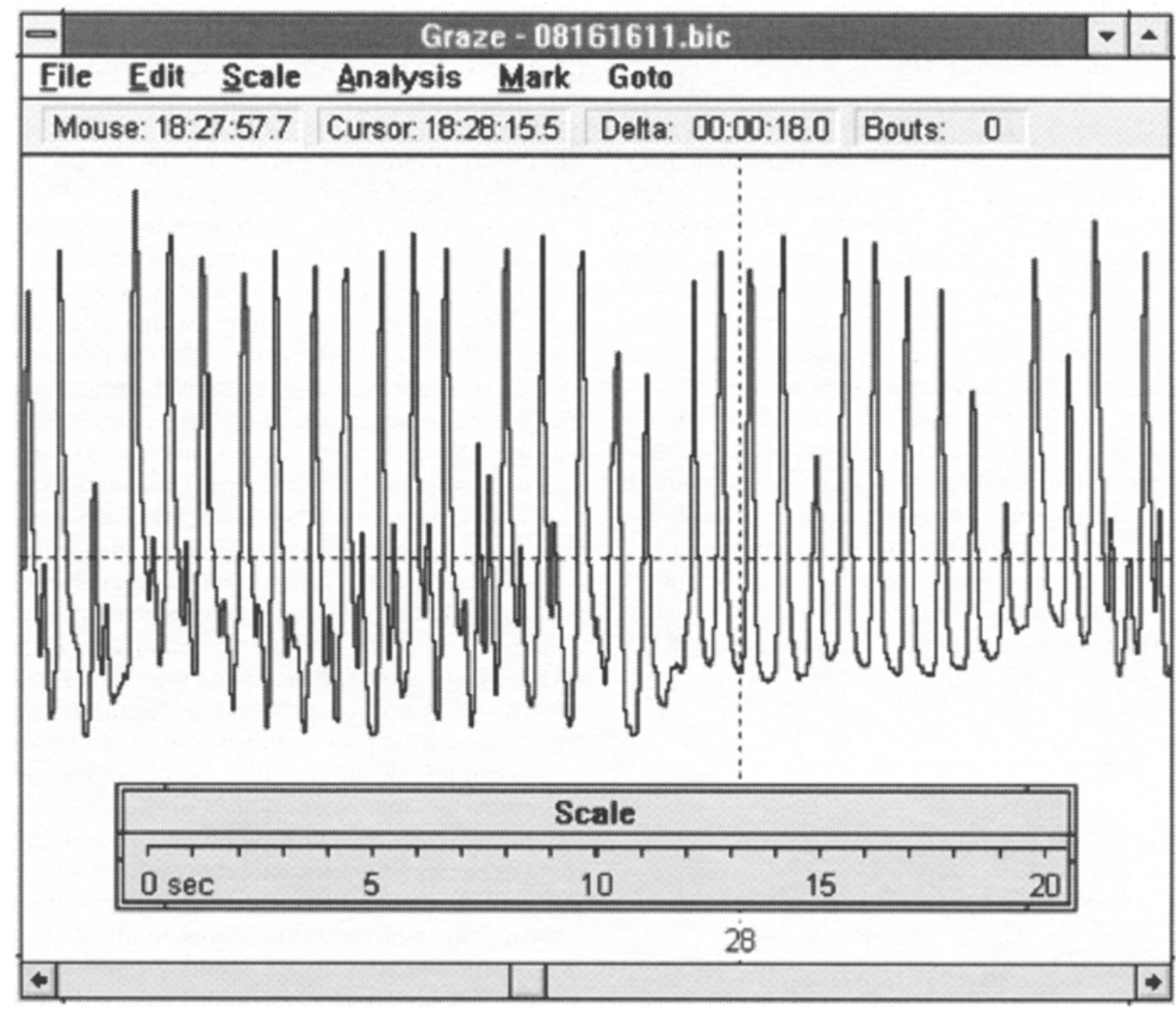

Figure 1. The main Graze window after a data file has been loaded. The main display area of the window plots the raw jaw movement amplitude data (vertical axis) against time (horizontal axis). 
ber, date, and time that the recording was started, etc.) using the Recording Info menu command.

\section{Identifying Jaw Movements}

The first stage in analyzing a jaw movement recording is to identify the jaw movements. Graze first searches through the raw waveform data and identifies all the peaks above a certain minimum amplitude (defined as the minimum prehension subpeak amplitude, described later). Graze measures the amplitude of a peak from the top of the peak down to the lowest trough associated with that peak. For example, Peak i (Figure 2) is measured down to the trough to the right of the peak (which is lower than the trough to the left), whereas Peak ii is measured down to the trough to the left of the peak (which is lower than the trough to the right).

Having identified all the peaks in the data file, Graze then uses two criteria to decide which peaks are jaw movements. For a peak to be categorized as a jaw movement, its amplitude has to be above a certain minimum jaw movement amplitude, and the interval between it and any other potential jaw movements has to be greater than a minimum inter-jaw-movement interval. The minimum jaw movement amplitude is specified in arbitrary units between 0 and 255, and the minimum jaw movement interval is specified in units of $1 / 20 \mathrm{sec}$. It is important to note that, although the user can change the criteria used to detect jaw movements, this applies globally to the whole data set-that is, different parameters cannot be set for individual jaw movements. Once Graze has established that a peak has an amplitude greater than the minimum jaw movement amplitude, it then establishes the interval between the peak and the immediately preceding jaw movement. If this interval is greater than the minimum jaw movement interval, the peak can be classified as a jaw movement. For example, assume that Peak i (Figure 3) has already been identified by Graze as a jaw movement. The next potential jaw movement is Peak ii. This meets the minimum jaw movement amplitude criterion (as discussed earlier), and the interval between Peaks i and ii (i.e., interval $a$ ) is greater than the minimum inter-jawmovement interval (10/20 sec, in this example). Peak ii can, therefore, be provisionally classified as a jaw movement. (The reason for this being a provisional classification should become apparent later.) The next potential jaw

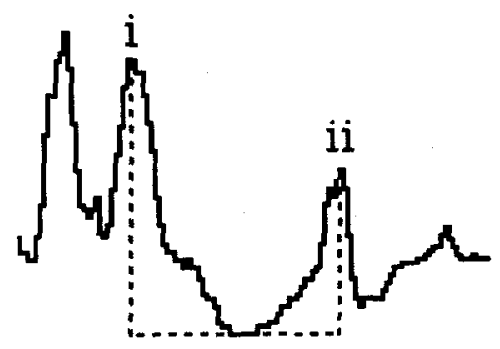

Figure 2. The amplitude of a peak is measured from the top of a peak to the lowest trough associated with that peak (e.g., the lowest trough for Peak $i$ is to the right of that peak, whereas the lowest trough for Peak ii is to the left).

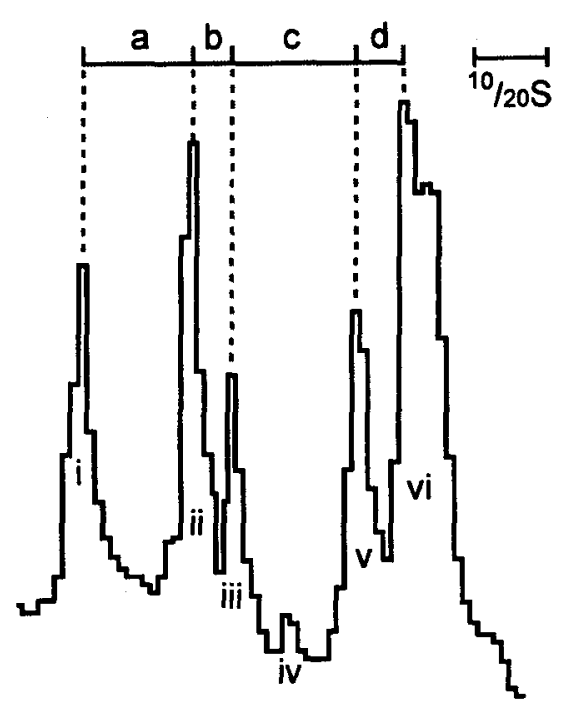

Figure 3. Graze uses amplitude and interval criteria to determine which peaks are jaw movements. See the main text for further explanation.

movement is Peak iii. However, although Peak iii meets the minimum jaw movement amplitude criterion, the interval between Peaks ii and iii (i.e., interval $b$ ) is less than the minimum inter-jaw-movement interval. Consequently, only one of the two peaks (i.e., either ii or iii) can be classified as a jaw movement. When deciding which peak to select as a jaw movement, Graze simply selects the largest-in this case, Peak ii. Peak ii, therefore, retains its provisional classification as a jaw movement, and Peak iii is rejected. Although the next potential jaw movement is Peak iv, this peak fails to meet the minimum jaw movement amplitude criterion (i.e., it is too small) and so is immediately rejected. The next potential jaw movement is Peak v. This meets the minimum jaw movement amplitude criterion. and the interval between Peak $v$ and the previous (albei1 provisional) jaw movement (i.e., Peak ii) is greater than the minimum inter-jaw-movement interval. Consequently. Peak $v$ can be provisionally classified as a jaw movement This also means that peak ii, which had previously beer provisionally classified as a jaw movement, can now be confirmed as a jaw movement, because Graze has nou established that there are no other potential jaw movements within the minimum inter-jaw-movement interval 0 : Peak ii. The final peak in the example data set that coulc be a potential jaw movement is Peak vi, which meets the minimum jaw movement amplitude criterion. However the interval between Peak vi and the previous jaw move ment $(v)$ is less than the minimum inter-jaw-movemen interval. Given that Peak vi has a greater amplitude thar Peak v, Peak vi is, therefore, provisionally classified as : jaw movement, and Peak $v$, although previously provi sionally classified as a jaw movement, is now rejected Graze has therefore identified three peaks (i.e., i, ii, anc vi) in the example data set as jaw movements.

Graze records a time associated with each jaw move ment. This is the sample number at which the jaw move 
ment waveform reaches its maximum value. This is the point at which the animal's gape reaches its maximumthat is, the point at which its jaws are open the widest. This part of the jaw movement event is used for timing purposes, because the peak maximum is virtually always well defined in the waveform - that is, as a sharp peak with a single maximum value sample. In contrast, the point at which the jaws are closed (i.e., the minimum value following a peak) was rarely well defined in the waveform - that is, the trough typically follows a shallow curve and has multiple minimum value samples. This characteristic is largely a result of the filtering used in the IGER Behavior Recorder, which is used to ensure that the jaw movement waveform returns to a fixed baseline when the jaw movement sensor is at rest.

When Graze has finished identifying jaw movements, the identified jaw movements are highlighted by drawing a line from the top of the window to the top of the jaw movement peak. This ensures that the user can quickly check exactly which peaks have been identified by Graze as jaw movements, allowing the user to check whether the jaw movement identification process has been accurate. Note that identified jaw movements cannot be individually edited or saved. If the user wishes to improve the accuracy of the jaw movement identification process, he or she needs to execute the Identify Jaw Movements menu command again, using different parameters, until he or she is satisfied with the results.

If the user subsequently needs to reanalyze a data file, he or she has to load the file back into Graze and then execute the Identify Jaw Movements menu command again. Note that the results summary files created at the end of data analysis (described later) include details of the jaw movement identification parameters used, allowing the user to generate an identical set of identified jaw movements, if required.

\section{Identifying Bouts}

Once the user is satisfied that the program has correctly identified the jaw movements, the next stage of the analysis is to identify bouts of jaw movements. A bout is defined as a series of $n$ or more jaw movements separated from any preceding or subsequent jaw movements by a maximum interval $(t)$. Although the user can specify both the minimum number of jaw movements per bout $(n)$ and the maximum intrabout interval $(t)$, the automatic discrimination between eating and ruminating (described later) requires the maximum intrabout interval to be fixed at $3 \mathrm{sec}$. This is to ensure that each bolus during ruminating is marked as a separate bout.

For example, suppose that bouts were automatically identified in the pattern of jaw movements shown in Figure 4, using an intrabout interval $(t)$ of $4 \mathrm{sec}$ and a minimum number of jaw movements per bout $(n)$ of 4 . The intervals between the first 5 jaw movements are all less than $4 \mathrm{sec}$, and consequently, these 5 jaw movements form a bout. As in Graze, the bout is shown by changing the background color behind the jaw movements that form the bout (as is shown in Figure 5). The interval between the 5th and the 6th jaw movements is greater than $4 \mathrm{sec}$, so the 6th jaw movement cannot join the first 5 in the first bout. Although the 6th, 7th, and 8 th jaw movements are all less then $4 \mathrm{sec}$ apart, there are only 3 of them in the group. Therefore, the 3 cannot form a bout (which must have at least 4 jaw movements). Although the interval between the 11 th and the 12th jaw movements is nearly $4 \mathrm{sec}$, the intervals between the 9th-13th jaw movements are all less than $4 \mathrm{sec}$, and consequently, they form a second bout. Finally, the interval between the 13th and the 14th jaw movements is greater than $4 \mathrm{sec}$, so this single jaw movement cannot form part of a bout.

\section{Identifying Bout Behaviour}

Once the program has automatically identified bouts of jaw movements, Graze then uses a scoring system to automatically discriminate between eating and ruminating bouts. Each bout is tested against a series of rules (Table 1) that change the score for that bout if the conditions described in the rule are met. The rules were developed by the author on the basis of his extensive experience in an-

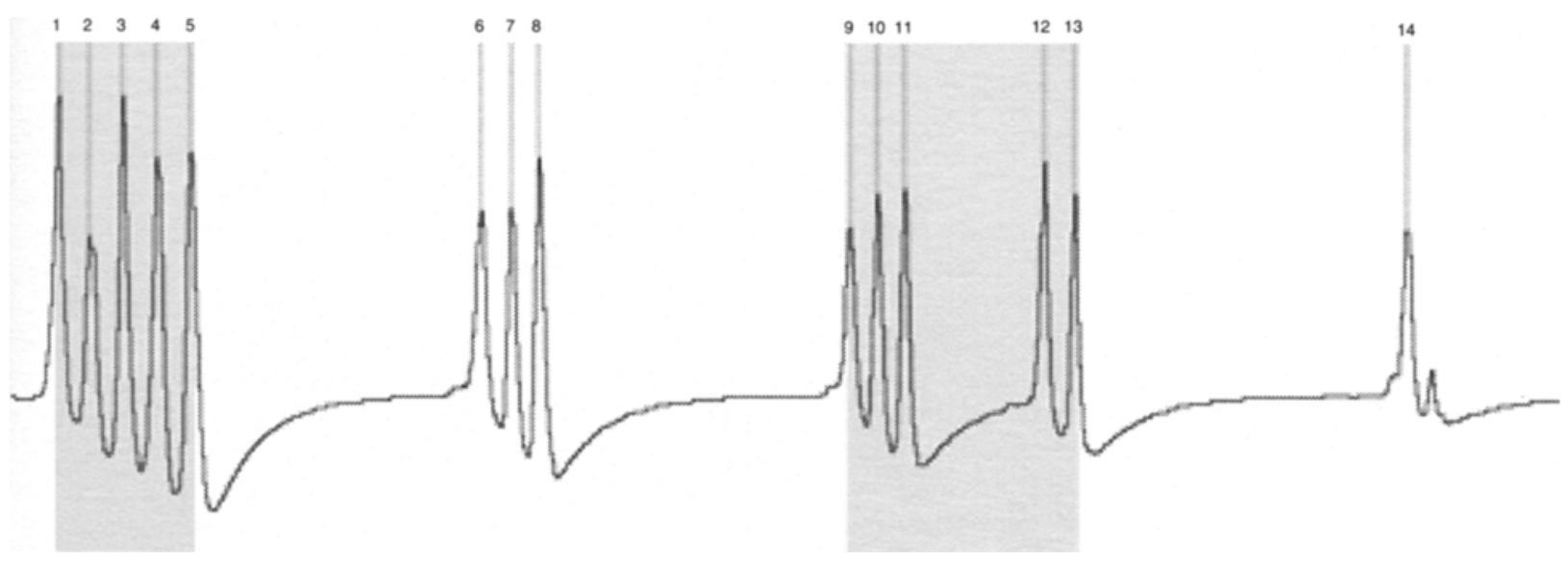

Figure 4. Graze uses jaw movement number and interval criteria to determine which groups of jaw movements form bouts. See the main text for further explanation. 


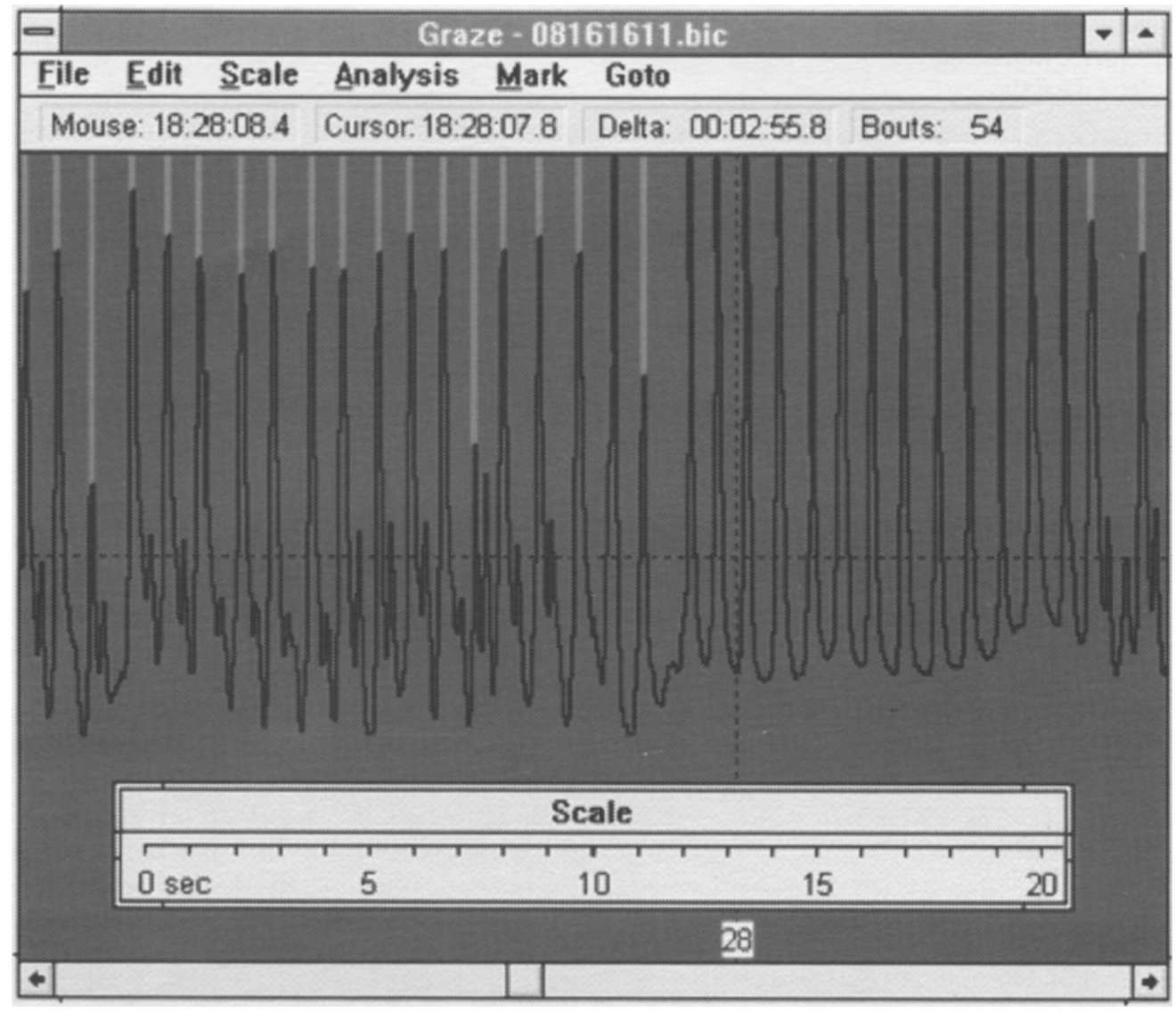

Figure 5. The main Graze window following data analysis. Jaw movements are shown by vertical lines from the top of the window to the top of the jaw movement waveform. Bouts (in this example, grazing') are shown by changing the background color behind the jaw movements that form each bout.

alyzing jaw movement recordings and have been shown to be empirically valid (see the Discussion section).

Each bout starts with a score of 0 - that is, undetermined. If, at the end of the test against the twelve rules, the bout still has a score of 0 , the bout remains as 'undetermined'. However, if the bout has a score greater than 0 but less than or equal to +2 , it is marked as "possibly ruminating." If the score is greater than +2 , it is marked as "probably ruminating." Similarly, a bout with a score of less than 0 but greater than or equal to -2 is "possibly grazing," and less than -2 is "probably grazing." The background color used to highlight bouts is changed to reflect the behavior category for each bout (as is shown in Table 2). The probably/possibly subcategories associated with eating and ruminating are intended to give the user some idea of the confidence that Graze has in its own automatic bout discrimination. Note that, when analyzing a data file (described later), Graze does not distinguish between the probably/possibly subcategories - that is, Graze simply distinguishes between grazing, ruminating, eating supplements, drinking (or other), and undetermined and ignores the possibly/probably subcategorization associated with eating and ruminating.
Note that, in the current version of Graze, the automatic bout behavior identification is limited to eating and ruminating. Other behaviors (such as eating supplements or drinking) are not detected automatically but can be marked manually, as will be described in the next section.

\section{Manually Editing Bouts}

As was described earlier, bouts of jaw movements are highlighted by changing the background color (Table 2) to indicate the behavior associated with each bout. This allows the user to quickly and easily check that Graze has correctly identified the appropriate behavior associated with each bout. If necessary, the user can manually edit bouts. The user can change the behavior associated with a bout by selecting the bout (by moving the cursor over it and pressing the mouse button) and then changing the behavior category, using the Mark menu command. Alternatively, the user can delete a bout by selecting it and then pressing the Delete key. The user is also free to mark their own bouts. To do this, the user moves the cursor to the desired start position of the bout and then presses the mouse button. This positions a flashing cursor at the start of the bout. The user can then move the cur- 
Table 1

The Twelve Rules Used to Discriminate Between Eating and Ruminating

\begin{tabular}{rlr}
\hline No. & \multicolumn{1}{c}{ Rule } & Score \\
\hline 1 & Peak amplitude variance low $(<400$ amplitude units $)$ & +1 \\
2 & Peak amplitude variance high $(>2000$ amplitude units $)$ & -1 \\
3 & Mean interpeak interval long $(>12 / 20 \mathrm{sec})$ & +1 \\
4 & Mean interpeak interval short $(<10 / 20 \mathrm{sec})$ & -1 \\
5 & Less than 10 jaw movements per bout & -1 \\
6 & Less than 20 jaw movements per bout \\
7 & More than 100 jaw movements per bout & -1 \\
8 & Peaks/jaw movements $>1.5$ & -2 \\
9 & Peaks/jaw movements $<1.2$ & -2 \\
10 & Undetermined or eating bout preceded and followed by & +2 \\
& ruminating bout within 10 sec & +2 \\
11 & $\begin{array}{l}\text { Ruminating bout neither preceded nor followed by any } \\
\text { other ruminating bouts }\end{array}$ & 0 \\
12 & $\begin{array}{l}\text { Ruminating bouts separated by at least } 10 \text { sec from any } \\
\text { preceding or following ruminating bouts }\end{array}$ & -2 \\
\hline
\end{tabular}

Note-If a bout meets the conditions of the rule, the score for that bout is changed as shown in the right-hand column. Peaks are all waveform peaks greater than the minimum prehension subpeak amplitude (and regardless of interpeak interval). Jaw movements are a subset of peaks that are greater than the minimum jaw movement amplitude and are separated by at least the minimum interjaw movement interval.

sor to the desired end position of the bout (scrolling the data window, if required) and press the mouse button while holding down the Shift key. This selects the portion of the recording that is to form the new bout and is indicated by inverting the colors used to display this portion of the recording. The user can then use one of the Mark menu commands to set the behavior category associated with the new bout. The facility to manually edit bouts allows the user to, for example, remove any bouts that are not of interest because they are outside the experimental measurement period.

Once the user is satisfied that all the bouts have been correctly identified, the many ruminating bouts can be joined together (concatenated) by using the Join Ruminating Bouts command. This joins adjacent ruminating bouts if the interval between them is less than a given duration, ensuring that each ruminating bout consists of a number of boli, rather than a single bolus per bout. Similarly, grazing bouts can be joined into meals by using the Join Grazing Bouts command, using a minimum intermeal interval criterion specified by the user. The joining of bouts (ruminating and/or eating) reduces the total number of bouts listed in the output files. Although this can simplify the subsequent analysis of the data, this is at the expense of some accuracy. By allowing the parameters used to join bouts together to be specified, Graze allows the user to make their own tradeoff between simplicity and accuracy. Joining ruminating bouts does not affect the way Graze counts boli. Graze counts the number of boli processed during ruminating bouts (whether or not they have been joined) by detecting the periodic intervals between boli when the animal is not chewing. The minimum interval used by Graze to detect boli during data analysis can be specified by the user in the Cattle Bout Analysis dialog box (described later).
Bout information can also be saved into a Bout Mark file using the Save Bout Mark File menu command. This allows the user to read the edited bout mark information back into Graze (using the Open Bout Mark File menu command) should he or she subsequently need to reanalyze a file.

\section{Identifying Jaw Movement Type}

The final stage in the analysis of the jaw movement waveform is discriminating between bites and chews. There are two characteristics that can be used to distinguish a bite from a chew in cattle. In the majority of animals, the main peak associated with a bite is followed by a small subpeak (e.g., the first 16 jaw movements in Figures 1 and 5 are followed by subpeaks). The exact biological reason why, during a bite, a subpeak is observed is not known, but it has been shown to be an empirically valid discriminator between bites and chews (Champion, Rutter, \& Orr, 1997). Graze searches through each grazing bout and identifies all the jaw movements that have a subpeak as bites. However, there can occasionally be a very small subpeak following chews. Consequently, only subpeaks greater than a minimum prehension subpeak threshold result in the jaw movement being categorized as a bite.

Occasionally, a cattle jaw movement recording will not contain any subpeaks following the bites. In this case, the shape of the jaw movement waveform must be used to distinguish between bites and chews. Bites show a fast rise-time, as compared with the fall time, whereas chews are more symmetrical in shape, with approximately equal rise-times and fall-times (Champion, Rutter, \& Orr, 1997). The Cattle Bout Analysis dialog box can be used to set the threshold for using the shape of the jaw movement to discriminate between bites and chews. This is the minimum rise:total ratio for mastication (\%) parameter, which has a default value of $40 \%$. This means that if the rise-time forms $40 \%$ or more of the total jaw movement duration, the jaw movement is classified as a chew; otherwise, it is a bite. Note that both the subpeak and the rise-time bite/chew discrimination processes can operate at the same time. However, if the recording clearly shows subpeaks after the bites, the user can effectively disable the rise time discrimination part of the process by setting "Min. rise:total ratio for mastication" to $1 \%$.

Once Graze has performed the bite/chew discrimination process, the colors of the lines used to mark the identified jaw movements during grazing bouts are changed

Table 2

Colors Used by Graze to Display Different Bout Types

\begin{tabular}{ll}
\hline Behavior Category & Bout Mark Color \\
\hline Probably ruminating & red \\
Possibly ruminating & light red \\
Probably grazing & green \\
Possibly grazing & light green \\
Eating supplements & light brown \\
Drinking or other & light blue \\
Undetermined & light gray \\
\hline
\end{tabular}


Table 3

An Example of an Analysis Results Summary File

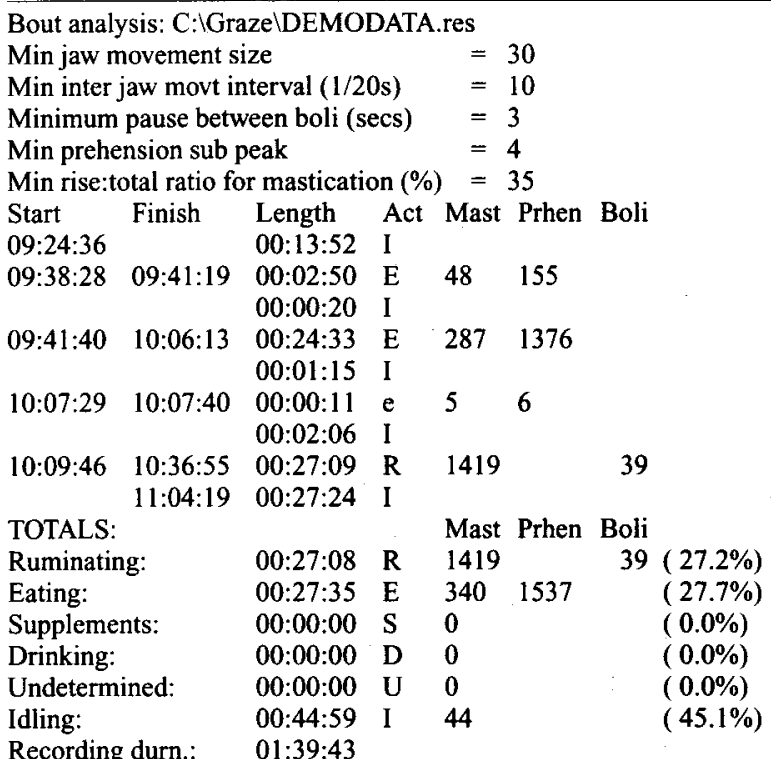

Note-The start of the file lists the parameters used to analyze the data. The next section lists relevant information about each bout: the start and finish time, the duration (in hours:minutes:seconds), the behavior activity code ("E" or "e" for grazing, " $R$ " or " $r$ " for ruminating, " $I$ " for idling, " $S$ " for eating supplements, " $D$ " for drinking, and " $U$ " for undetermined), and finally, the numbers of mastications ("Mast") and bites ("Prhen") for grazing bouts or the numbers of mastications ("Mast") and "Boli" processed during ruminating. The final section of the file gives the total time (in hours:minutes:seconds, as well as the percentage of the total recording) spent performing each activity, along with the relevant jaw movement and boli counts.

to light green for a bite and to blue for a chew, as is shown in Figure 5.

\section{Summarizing the Analysis Results}

At the end of the analysis, the user has the option of saving a summary of the analysis results. This can be as a simple text file (an example of which is shown in Table 3) or as a Comma Separated Values file that can be read directly into a spreadsheet. These files give the parameters used to analyze the file, a bout-by-bout summary (giving bout start and finish times, durations, behavior activity, jaw movement counts, etc.), and a summary of the time spent performing each of the main behavior categories. These summary data can then be used in statistical packages when analyzing the experiment as a whole.

\section{DISCUSSION}

The IGER Behavior Recorder and Graze have been validated as a whole system for recording and analyzing the eating and ruminating behavior of sheep (Rutter, Champion, \& Penning, 1997). The automatic system was compared with manual observation, and there was an overall index of concordance of $91.0 \%$ between the two methods in identifying periods of eating and ruminating. Rutter, Champion, and Penning concluded that "the au- tomatic system was at least as accurate as manual observation" (p. 192). The ability to discriminate between bites and chews in cattle was tested by Champion, Rutter, and Orr (1997). Although there was a high mean square prediction error (MPE) between manual observation and the automatic system in some animals, the MPE between the manual and the automatic systems was less than that between the two observers for most animals. This led Champion, Rutter, and Orr to conclude that the automatic system "provided a more accurate method of identifying bites taken by cattle during grazing than manual observation" (p. 172). Consequently, Graze forms an important part of a powerful research tool for ruminant foraging behavior studies.

As with most software, Graze is frequently updated to incorporate new features. The next version of the program (currently under development) will include the ability to analyze lying, standing, and walking behavior and will create analysis results files that can be read directly into the Observer behavior analysis system (Noldus, 1991). The bite/chew discrimination algorithms are also being modified and validated for use with sheep jaw movement recordings.

Although Graze was developed for use with the IGER Behavior Recorder, the program could be used to analyze recordings from other systems (assuming that the jaw movements were recorded by using a similar technique and at a similar resolution and frequency). The binary behavior data file format used by Graze is published in Rutter, Champion, and Penning (1997), allowing data from other systems to be converted into a format that could be read by Graze.

\section{REFERENCES}

Champion, R. A., Rutter, S. M., \& OrR, R. J. (1997). Distinguishing bites and chews in recordings of the grazing jaw movements of cattle. In Proceedings of the Fifth Research Conference of the British Grassland Society. Reading, U.K.: British Grassland Society.

Champion, R. A., Rutter, S. M., \& Penning, P. D. (1997). An automatic system to monitor lying, standing and walking behaviour of grazing animals. Applied Animal Behaviour Science, 54, 291-305.

HoDGSON, J. (1977). Factors limiting herbage intake by the grazing animal. In Proceedings of the International Meeting on Animal Production from Temperate Grassland (pp. 70-78). Reading, U.K.: British Grasslands Society.

Noldus, L. P. J. J. (1991). The Observer: A software system for collection and analysis of observational data. Behavior Research Methods, Instruments, \& Computers, 23, 415-429.

PEnNing, P. D. (1983). A technique to record automatically some aspects of the grazing and ruminating behavior in sheep. Grass \& Forage Science; 38, 89-96.

Penning, P. D., Steel, G. L., \& Johnson, R. H. (1984). Further development and use of an automatic recording system in sheep grazing studies. Grass \& Forage Science, 39, 345-351.

RutTer, S. M., Beresford, N. A., \& RoberTs, G. (1997). Use of GPS to identify the grazing areas of hill sheep. Computers \& Electronics in Agriculture, 17, 177-188.

Rutter, S. M., Champion, R. A., \& Penning, P. D. (1997). An automatic system to record foraging behavior in free-ranging ruminants. Applied Animal Behaviour Science, 54, 185-195.

(Manuscript received December 18, 1998; revision accepted for publication October 3, 1999.) 